\title{
Antibacterial quality of some antibiotics available in five administrative areas along the national borders of Tanzania
}

This article was published in the following Dove Press journal:

Integrated Pharmacy Research and Practice

8 December 2014

Number of times this article has been viewed

\section{Kennedy D Mwambete}

Department of Pharmaceutical Microbiology, Muhimbili University of Health and Allied Sciences, Dar es Salaam, Tanzania
Correspondence: KD Mwambete Department of Pharmaceutical Microbiology, Muhimbili University of Health and Allied Sciences (MUHAS), PO Box 65013, Dar es Salaam, Tanzania Tel +255787508782

Fax +25522 2150465

Email kmwambete@muhas.ac.tz
Introduction: In developing countries like Tanzania, bacterial infections are becoming increasingly difficult to treat with available antibiotics. Poor quality antibiotics jeopardize the management of bacterial infections and contribute to the development of antibiotic resistance. Poor storage and harsh tropical climatic conditions accelerate deterioration of antibiotics. Hence, this study investigated the antibacterial effect of antibiotics available in five administrative regions along the national borders of Tanzania.

Materials and methods: A cross-sectional study involved the purchase of antibiotics from the Mwanza, Arusha, Kilimanjaro, Mbeya, and Kagera administrative regions. The Kirby-Bauer disk diffusion method was employed to assess antibacterial effects of the antibiotics against Salmonella typhi, Klebsiella spp., Staphylococcus aureus, Pseudomonas aeruginosa, and Escherichia coli. Inhibition zones (IZ) were determined as previously described. Analysis of variance was used to examine the IZ measured using test antibiotics to their respective control antibiotics; differences were considered significant at $P<0.05$.

Results: Seventy-six antibiotic samples from 22 manufacturers were tested. Six antibiotic samples were from anonymous manufacturers and 29 antibiotic samples had no manufacturing or expiration dates. Different samples of the same antibiotics produced variable results. IZ measured using different samples of ampicillin (AMP) and ciprofloxacin and their control antibiotics revealed significant differences when tested against $S$. typhi $(P<0.05)$. Samples of tetracycline and chloramphenicol resulted in IZ comparable to their controls against $S$. typhi. All samples of AMP yielded comparable IZ on Klebsiella spp., whereas samples of chloramphenicol and tetracycline exerted IZ against $P$. aeruginosa that were not statistically different from their respective control antibiotics $(P>0.05)$. Ambiguous antibacterial profiles were exhibited by samples of AMP, chloramphenicol, cotrimoxazole, and amoxicillin as compared to their respective control antibiotics.

Conclusion: Differences in antibacterial effects were found among samples of the same type of antibiotic. Results suggest the existence of counterfeit and/or substandard drugs in Tanzania.

Keywords: antibiotics, antibiotic resistance, counterfeit antibiotics

\section{Introduction}

Counterfeit medications are a global health concern. ${ }^{1,2}$ When these include counterfeit antimicrobial agents, the problem becomes a major threat to public health with many devastating consequences for patients, including the emergence of antimicrobial resistance, and increased morbidity and mortality. ${ }^{3,4}$ Physicians who prescribe counterfeit antimicrobial agents/antibiotics lose their credibility to patients. ${ }^{5}$ As many as $50 \%$ of the medicines sold in Africa and 30\% sold in Southeast Asia and Latin America 
might be counterfeit. ${ }^{6}$ Therefore, not only do patients have to worry about their illnesses, but they must also consider the possibility that they are being treated with counterfeit medications. In Africa, over $75 \%$ of antibiotics are alleged to be inappropriately prescribed and the consequences fall primarily on poor populations that are not covered by health insurance policies. ${ }^{7}$

Over the past decade, there has been an increase in public awareness of the existence of counterfeit and substandard drugs. ${ }^{2,8-10}$ Drug regulations are known to be ineffective in developing countries. ${ }^{11-13}$ Many pharmaceutical products are a problem, but the existing data suggest that antimicrobial agents, particularly antibiotics, are the most often counterfeited products. Studies indicate that a country's capacity to restrict availability of dangerous or substandard medicines depends on its wealth. ${ }^{14}$ It is surprising that about $30 \%$ of the World Health Organization (WHO) member countries have poor mechanisms for controlling counterfeit medications. ${ }^{10}$

Much of the current discourse on infectious diseases and antibiotic resistance as it affects sub-Saharan Africa is limited to the pressing problems associated with emerging and re-emerging antibiotic-resistant microorganisms. ${ }^{15,16}$ Antibiotic resistance compromises the management of common bacterial infections, including respiratory and sexually transmitted infections, and diseases spread by the fecal-oral route and other diarrheal diseases, which are the major health challenges in most resource-limited countries. ${ }^{3,17}$

Antibiotic resistance is a natural phenomenon that occurs whenever antibiotics are in use.$^{18}$ However, there are human behaviors that contribute to the rapid development and spread of bacterial antibiotic resistance, including easy availability of antibiotics to patients without prescriptions. ${ }^{19,20}$ Bacteria are efficient at enhancing antibiotic resistance, not only because of their ability to multiply rapidly, but also because they can transfer their antibiotic resistance genes during replication. ${ }^{16}$ Antibiotic-resistant microorganisms will not be readily affected by an antimicrobial agent during a regular course of treatment. Antibiotic-resistant bacteria can pass their antibiotic resistance genes to other bacteria through "conjugation", whereby plasmids carrying these genes are transferred from one microorganism to another. ${ }^{21}$ Resistance to a single drug can thus spread rapidly through bacterial populations. When antimicrobial agents, including antibiotics, are used incorrectly, for too little a time, at an inadequate dose, or when the wrong agent is selected to treat an infection, microorganisms have an opportunity to adapt and replicate. ${ }^{22}$

To counteract the threat of antibiotic resistance, which is attributable to the high prevalence of counterfeit and substandard antibiotics, it is important to understand the magnitude of the problem. Few studies have systematically examined poor quality antimicrobial drugs to assess the quantity of active ingredients present. ${ }^{23,24}$ Although the drugs used to treat serious diseases such as malaria, HIV/AIDS, tuberculosis, or other infections are often counterfeit, there have been few reports on the characteristics of low-quality antimicrobial drugs. Therefore, the goal of this study was to assess the antibacterial quality of antibiotics that are available in five administrative regions along the border of Tanzania: Arusha; Kilimanjaro; Mbeya; Mwanza; and Kagera.

\section{Materials and methods Geographical regions examined and study design}

The study included the Mbeya, Arusha, Kilimanjaro, Mwanza, and Kagera administrative regions of Tanzania and involved any drug outlets (pharmacies, drugs stores, and Accredited Drug Dispensing Outlets) in towns and cities. The regions selected are national border regions of Tanzania and some regions have borders with more than one neighboring country and are thus prone to the presence of unauthorized and/or unregistered medicines in the marketplace (Figure 1). Moreover, some of these regions comprise the most ancient, commercial, and cosmopolitan cities in Tanzania.

\section{Sampling procedure}

A probability sampling technique (cluster sampling) was employed. Two-stage cluster sampling entailed division of the study population into clusters, and random samples were selected from the clusters. The clusters were organized by localities within each administrative region. After clusters were selected, units/pharmacies within the clusters were randomly selected. No units/pharmacies from clusters that were not selected were included in the study. A sample size of 15 different brands of antibiotics (in solid dosage forms, tablets, and/or capsules) was targeted based on the following assumption: at least 15 different brands of antibiotics were available in the market in each of the five regions. The antibiotics were purchased randomly, although one antibiotic could be purchased more than twice, and both were assayed separately to compare the results among manufacturers or sales premises and localities.

\section{Biological and chemical materials}

Reference strains of bacteria Salmonella typhi (CT18), Klebsiella pneumoniae (ATCC700603), Staphylococcus aureus (ATCC25923), Pseudomonas aeruginosa 


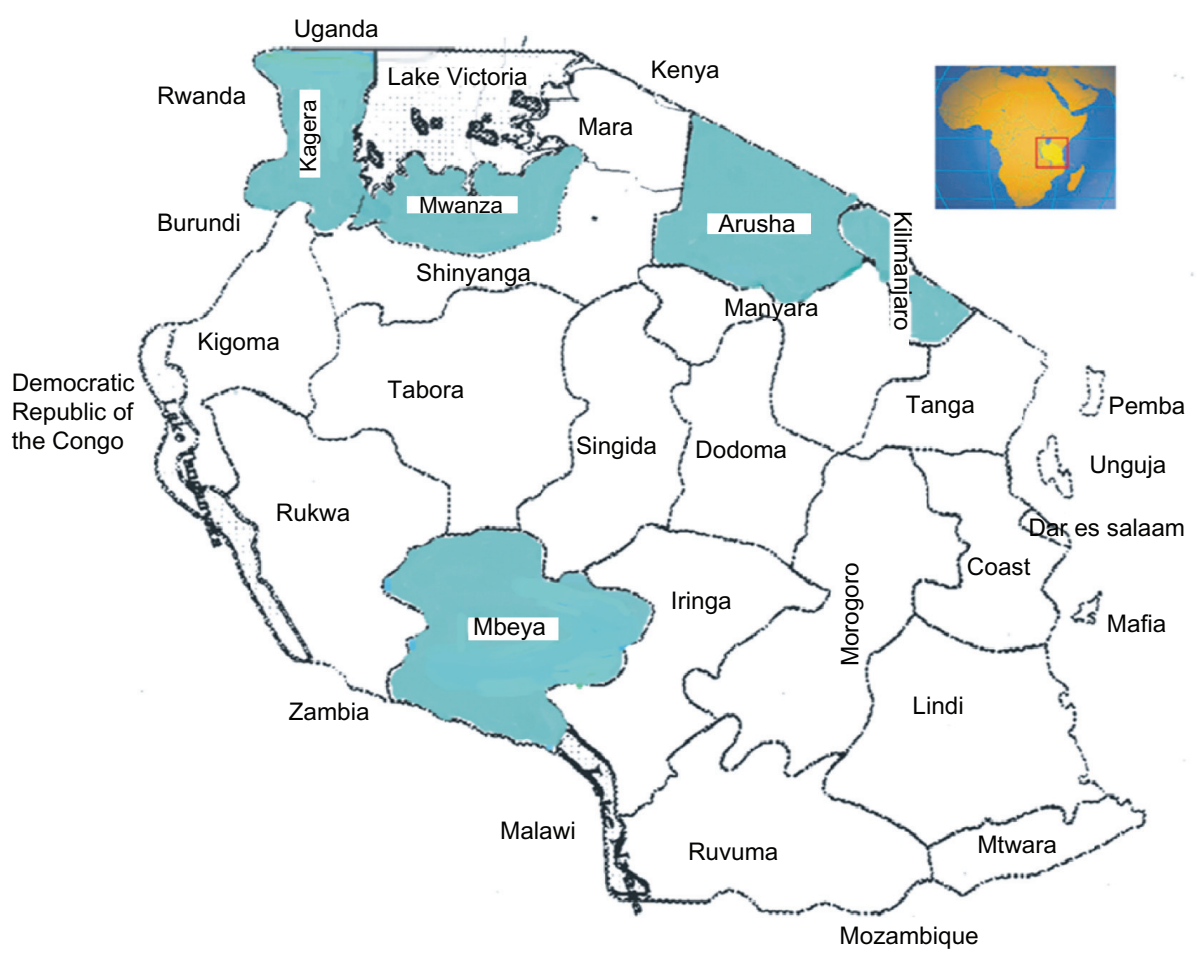

Figure I Map of Tanzania showing the five administrative regions (shaded) studied.

(ATCC27853), and Escherichia coli (ATCC25922) were obtained from the Microbiology/Immunology Laboratory at School of Medicine (MUHAS). The antibiotics purchased from the above mentioned regions were tested for their antibacterial effects (Tables 1 and 2). The test antibiotics were randomly purchased from pharmacies, medical stores, and Accredited Drug Dispensing Outlet shops located within the five study regions. Standard antibiotic sensitivity disks were purchased and used as positive controls.

\section{Preparation of test antibiotic and control antibiotic susceptibility disks}

The antibiotic samples purchased were weighed and dissolved in sterile distilled water and embedded into $5.0 \mathrm{~mm}$ Whatman ${ }^{\circledR}$ No 1 filter paper disks. The antibiotic sensitivity disks were prepared as per the Clinical Laboratories Standards Institute ${ }^{25}$ guidelines to contain the concentrations (between $5 \mu \mathrm{g}$ and $30 \mu \mathrm{g}$ ) equivalent to their respective standards (commercial standard antibiotic disks). The antibiotic (250 mg) was dissolved in $10 \mathrm{~mL}$ of sterile distilled water and a 1:10 dilution was prepared to yield a $2,500 \mu \mathrm{g} / \mathrm{mL}$ concentration. One-hundred Whatman No 1 filter paper disks were submerged in the solution with the assumption that each disk would contain $25 \mu \mathrm{g}$ of the antibiotic. Similar procedures were used to prepare all antibiotics.

The control antibiotic disks were commercially available antibiotic susceptibility disks: ampicillin (AMP;
$10 \mu \mathrm{g}$ ); amoxicillin/clavulanate (AMCL; $30 \mu \mathrm{g}$ ); amoxicillin (AMO; $25 \mu \mathrm{g}$ ); chloramphenicol (CHR; $30 \mu \mathrm{g}$ ); ciprofloxacin (CIP; $5 \mu \mathrm{g})$; cotrimoxazole (CTX; $25 \mu \mathrm{g})$; erythromycin (ERY; $15 \mu \mathrm{g}$ ); tetracycline (TET; $30 \mu \mathrm{g}$ ); and gentamicin (GEN; $10 \mu \mathrm{g})$, which served as positive controls. Each standard/control antibiotic disk was placed centrally on the same agar plate with a corresponding test antibiotic, and both were assayed against the reference strains of bacteria. A Whatman filter paper disk impregnated with sterile distilled water served as the negative control. After an overnight incubation at $37^{\circ} \mathrm{C}$, the inhibition zones (IZ) were determined and recorded as previously described..$^{26,27}$

\section{Antibacterial assays}

The antibiotic effects of different antibiotic samples purchased were evaluated against reference strains of bacteria using the Kirby-Bauer disk diffusion method. Five discrete colonies of the reference strains of bacteria were separately inoculated into $5 \mathrm{~mL}$ of culture broth and incubated at $37^{\circ} \mathrm{C}$ for $4-6$ hours. The resultant bacterial suspensions were adjusted to match a standard turbidity (McFarland; $0.5 \mathrm{M}$ ) prior to subjecting them to susceptibility profiling on Mueller-Hinton agar plates (Difco ${ }^{\mathrm{TM}}$; BD, Franklin Lakes, NJ, USA) as per the Clinical Laboratories Standards Institute recommendations. ${ }^{25,27}$

The prepared antibiotic disks $(5-30 \mu \mathrm{g}$, as described above) were placed aseptically onto the agar plate using 
Table I Antibiotics from the Kagera, Arusha, and Kilimanjaro administrative regions of Tanzania

\begin{tabular}{|c|c|c|c|c|c|c|}
\hline Region & Locality & Drug origin & Drug & Drug batch number & Manufacturing date & Expiration date \\
\hline \multirow[t]{16}{*}{ Kagera } & Bukoba Town & India & AMP & AGOII08 & July II & July I4 \\
\hline & & India & ERY & BI69MIO & - & - \\
\hline & & Tanzania & $\mathrm{AMCL}$ & CLI005MI2 & - & - \\
\hline & & India & CTX & CZFBOII6 & - & - \\
\hline & & India & CIP & KD525 & November 10 & October 13 \\
\hline & & Tanzania & ERY & PCOI26 & - & - \\
\hline & & India & CTX & T21045 & - & - \\
\hline & & Tanzania & AMP & 120010 & January 12 & December 14 \\
\hline & Mtukula & India & ERY & I80M02 & - & - \\
\hline & & India & CIP & 209 & November II & October 14 \\
\hline & & Kenya & CTX & $2 \mathrm{~A} 185$ & - & - \\
\hline & & Kenya & CIP & $2 \mathrm{~B} 77$ & May II & April 14 \\
\hline & & Tanzania & $\mathrm{AMCL}$ & 56487 & August II & August 14 \\
\hline & & India & CIP & GL3206 & - & - \\
\hline & & India & CIP & GL3207 & January 10 & October 13 \\
\hline & & Kenya & GEN & 100417 & April 10 & March 13 \\
\hline \multirow[t]{4}{*}{ Arusha } & Arusha City & Tanzania & CIP & 110024 & January II & January 15 \\
\hline & & India & CIP & CB03FII & January II & May 14 \\
\hline & & Tanzania & ERY & 110008 & - & - \\
\hline & & India & TET & 120010 & - & - \\
\hline \multirow[t]{21}{*}{ Kilimanjaro } & Moshi Town & Unknown & $\mathrm{CHR}$ & 12009 & July 12 & March 14 \\
\hline & & Kenya & CTX & 11147 & September II & August 15 \\
\hline & & India & AMP & $262 \mathrm{MIOII}$ & - & - \\
\hline & & India & $\mathrm{AMCL}$ & AGOI5M7 & April 12 & March 14 \\
\hline & & Unknown & ERY & $\mathrm{C} 21012$ & May 12 & April 14 \\
\hline & & India & GEN & M005I7 & September II & August 14 \\
\hline & & India & GEN & M91215 & September 09 & December 12 \\
\hline & & Tanzania & ERY & 91111 & - & - \\
\hline & & India & GEN & 91215 & December 09 & December 12 \\
\hline & & People's Republic of China & GEN & 110317 & March II & February 14 \\
\hline & & People's Republic of China & TET & 110502 & - & - \\
\hline & & India & CTX & $|2028|$ & - & - \\
\hline & & India & ERY & 121057 & - & - \\
\hline & & India & CIP & $240 \mathrm{M} 02$ & - & - \\
\hline & Mkuu & Unknown & TET & L023 & February II & April 14 \\
\hline & & India & CTX & CZFBOI39 & June 12 & July 15 \\
\hline & & India & CTX & CZFBI6I & June 12 & June 15 \\
\hline & Rombo & Tanzania & ERY & CJ2004 & July 12 & March I4 \\
\hline & & India & $\mathrm{AMCL}$ & SCI29 & March 12 & February 14 \\
\hline & & People's Republic of China & $\mathrm{CHR}$ & 101123 & February II & July 14 \\
\hline & & Tanzania & CIP & 120007 & January 12 & November 14 \\
\hline
\end{tabular}

Abbreviations: AMP, ampicillin; ERY, erythromycin; AMCL, amoxicillin/clavulanate; CTX, cotrimoxazole; CIP, ciprofloxacin; GEN, gentamicin; TET, tetracycline; CHR, chloramphenicol.

sterile forceps. The disks were placed $24 \mathrm{~mm}$ apart and at least $15 \mathrm{~mm}$ from the edge of the Petri dish. The plates were incubated at $37^{\circ} \mathrm{C}$ for 24 hours. Each sample of antibiotic was tested twice in triplicate. Results were expressed as the diameter of IZ produced by the reference bacterial strains tested with the antibiotics purchased or the control (commercial susceptibility antibiotic disks), as previously described. ${ }^{27}$

\section{Ethical issues and definition of terms}

The University Ethical Committee approved this study. For legal and commercial purposes, the antibiotic manufacturers were not revealed, and only the antibiotic sample batch numbers and their country of origin are given.

\section{Statistical analysis}

Data were analyzed using the IBM SPSS statistics 20 computer package for analysis of variance of the IZ (antibacterial effect) exerted by each test antibiotic (batch) on each of the reference bacteria. The Dunnett's test (two-sided) was used to compare the level of significant differences between each sample with its control antibiotic with respect to the observed IZ. Differences in IZ among the samples and 
Table 2 Antibiotics from Mwanza and Mbeya administrative regions of Tanzania

\begin{tabular}{|c|c|c|c|c|c|c|}
\hline Region & Locality & Drug origin & Drug & Drug batch number & Manufacturing date & Expiration date \\
\hline \multirow[t]{18}{*}{ Mwanza } & Magu & India & CTX & 101685 & - & - \\
\hline & & India & CIP & 298M03 & February 12 & February 15 \\
\hline & & India & CTX & B505 & April 12 & February 15 \\
\hline & & India & AMO & CD50II004-A & - & - \\
\hline & & India & CTX & 101693 & - & - \\
\hline & & India & AMO & 1034-C & - & - \\
\hline & Sengerema & Kenya & TET & $2 B 358$ & May II & April 14 \\
\hline & & India & CIP & B238M02 & June 12 & July I5 \\
\hline & & People's Republic of China & GEN & M07I49 & July II & June 14 \\
\hline & Igombe & Unknown & $\mathrm{CHR}$ & CW200I & May 12 & April I5 \\
\hline & & Tanzania & $\mathrm{CHR}$ & 110022 & April II & April 14 \\
\hline & Igoma & Kenya & GEN & |2054| & May 12 & May 15 \\
\hline & Mwanza City & Tanzania & AMO & 100115 & April 10 & April 13 \\
\hline & & People's Republic of China & ERY & $|1073|$ & - & - \\
\hline & & People's Republic of China & GEN & 110826 & January II & December 13 \\
\hline & Pansiansi & India/Cyprus & CIP & B300 & November II & November II \\
\hline & & Tanzania & $\mathrm{AMCL}$ & $\mathrm{SCI} 16$ & January 12 & December 13 \\
\hline & & Kenya & CTX & $2 \mathrm{~A} 168$ & May II & December 15 \\
\hline \multirow[t]{17}{*}{ Mbeya } & Mbozi & India & CTX & CZFOI49 & February II & January 15 \\
\hline & & India & CTX & MRAGII4 & November 10 & February 15 \\
\hline & & Tanzania & CTX & TX1013 & January II & January 13 \\
\hline & Tunduma & Unknown & ERY & 52001 & - & - \\
\hline & & India & CIP & 208MII & November 10 & November 14 \\
\hline & & India & AMP & CA036MIO & - & - \\
\hline & & Tanzania & TET & 110007 & April II & April I4 \\
\hline & & Tanzania & AMP & 120014 & January 12 & January 14 \\
\hline & & Kenya & TET & $\mathrm{BI} 78$ & - & - \\
\hline & Kyela & India & CTX & CZFBOI57 & - & - \\
\hline & & India & CTX & MRAG027 & - & - \\
\hline & & India & CTX & 415 & January II & February 15 \\
\hline & & India & CTX & 499M02 & January 12 & January 15 \\
\hline & Mbeya City & India & CIP & ВI43M03 & - & - \\
\hline & & India & CTX & MRAGI05 & - & - \\
\hline & & India & CTX & MRAGI52 & November 10 & October 15 \\
\hline & & India & CIP & $\mathrm{G} / 25 / 17 / 5$ & - & - \\
\hline
\end{tabular}

Abbreviations: CTX, cotrimoxazole; CIP, ciprofloxacin; AMO, amoxicillin; TET, tetracycline; GEN, gentamicin; CHR, chloramphenicol; ERY, erythromycin; AMCL, amoxicillin/clavulanate; AMP, ampicillin.

between the samples and their respective control antibiotics were considered significant at $P<0.05$.

\section{Results}

\section{Distribution of antibiotic samples by regions and manufacturers}

Seventy-six different samples of antibiotics from 22 pharmaceutical industries/manufacturers were purchased from pharmacies and drug stores in the Mbeya, Mwanza, Kagera, Kilimanjaro, and Arusha administrative regions of Tanzania (Figure 1 and Tables 1 and 2) and tested for their antibacterial effects. Six samples were from anonymous manufacturers and 29 samples had no indication of their manufacturing or expiration dates (Tables 1-3). The six samples (two ERY, two CHR, one TET, and one ERY) had concealed countries of origin (Tables 1 and 2). One-quarter of the samples tested comprised CTX (number $[\mathrm{n}]=20 ; 26.3 \%$ ) and the least number of samples were AMO $(n=3 ; 3.9 \%)$ as shown in Table 3 . The samples were tested solely based on their availability during the study period.

\section{Antibacterial effects of antibiotics tested against reference bacteria Salmonella typhi}

Assessment of IZ of 15 different samples of CIP from five manufacturers tested against $S$. typhi revealed significant differences $(P=0.001 ; F=19.021$; degrees of freedom $[d f]=14)$. The IZ produced by each sample of CIP showed significant differences $(P<0.001)$ as compared to the results with the control antibiotic (control-CIP). Samples of GEN 
Table 3 Drugs, manufacturers, and samples

\begin{tabular}{lll}
\hline Drug & $\begin{array}{l}\text { Number of manufacturers } \\
\mathbf{N}=\mathbf{2 2}\end{array}$ & $\begin{array}{l}\text { Number of samples } \\
\mathbf{N}=\mathbf{7 6}(\%)\end{array}$ \\
\hline CIP & 5 & $15(19.7)$ \\
AMP & 4 & $5(6.6)$ \\
GEN & 5 & $8(10.5)$ \\
AMO & 2 & $3(3.9)$ \\
AMCL & 4 & $5(6.6)$ \\
CTX & 6 & $20(26.3)$ \\
ERY & 8 & $11(13.2)$ \\
TET & 5 & $6(7.9)$ \\
CHR & 4 & $4(5.3)$ \\
\hline
\end{tabular}

Abbreviations: N, number; CIP, ciprofloxacin; AMP, ampicillin; GEN, gentamicin; AMO, amoxicillin; AMCL, amoxicillin/clavulanate; CTX, cotrimoxazole; ERY, erythromycin; TET, tetracycline; CHR, chloramphenicol.

exhibited statistically significant differences in IZ ( $P=0.0001$; $F=6.990 ; d f=7)$, as shown in Figure 2. However, only two of eight samples tested revealed significant differences in IZ with respect to the control-GEN $(P<0.05)$. AMP samples were equally effective $(P=0.108 ; F=1.984 ; d f=4)$, but significant differences in their IZ were evident with respect to that of control-AMP $(P<0.05)$.

Antibacterial effects (IZ) of AMCL samples from five manufacturers (Table 3 ) showed no significant differences among them ( $P=0.019 ; F=3.942 ; d f=4)$, and none of the samples exhibited significant differences $(P>0.05)$ in IZ with respect to the control-AMCL (Figure 3). All three samples of AMO exhibited significant differences in IZ among them $(P=0.001 ; F=248.00 ; d f=2)$. Two of the three samples produced significantly different IZ compared to that of control-AMO ( $P=0.027$ and $P=0.005$ ). Three of eleven samples of ERY (one from India and two from Tanzania) revealed significant differences in IZ with respect to the control-ERY $(P<0.001)$. IZ of samples of ERY on $S$. typhi showed significant differences among them $(P=0.0001$; $F=39.132 ; d f=10)$. Only two of six samples of TET produced IZ that were significantly different from those of the control-TET $(P<0.05)$. ANOVA revealed no significant differences in IZ among samples of TET $(P=0.146 ; F=1.820$; $d f=5)$, as shown in Figure 4. Likewise, no significant differences were revealed among the four samples of CHR tested ( $P=0.507 ; F=0.885 ; d f=3)$. Results of CHR samples were different from those with the control-CHR $(P<0.05)$. The IZ of 20 samples of CTX from six manufacturers (Table 3) showed significant differences in IZ among them ( $P=0.0001 ; F=99.216 ; d f=19)$. Nevertheless, no apparent differences were observed, as shown in Figure 3, since only four of 20 samples exhibited significant differences in IZ compared to the control-CTX $(P=0.0001 ; 0.005$; and 0.011 [two samples]) and, of these, three samples of drugs were from India and one was from Kenya.

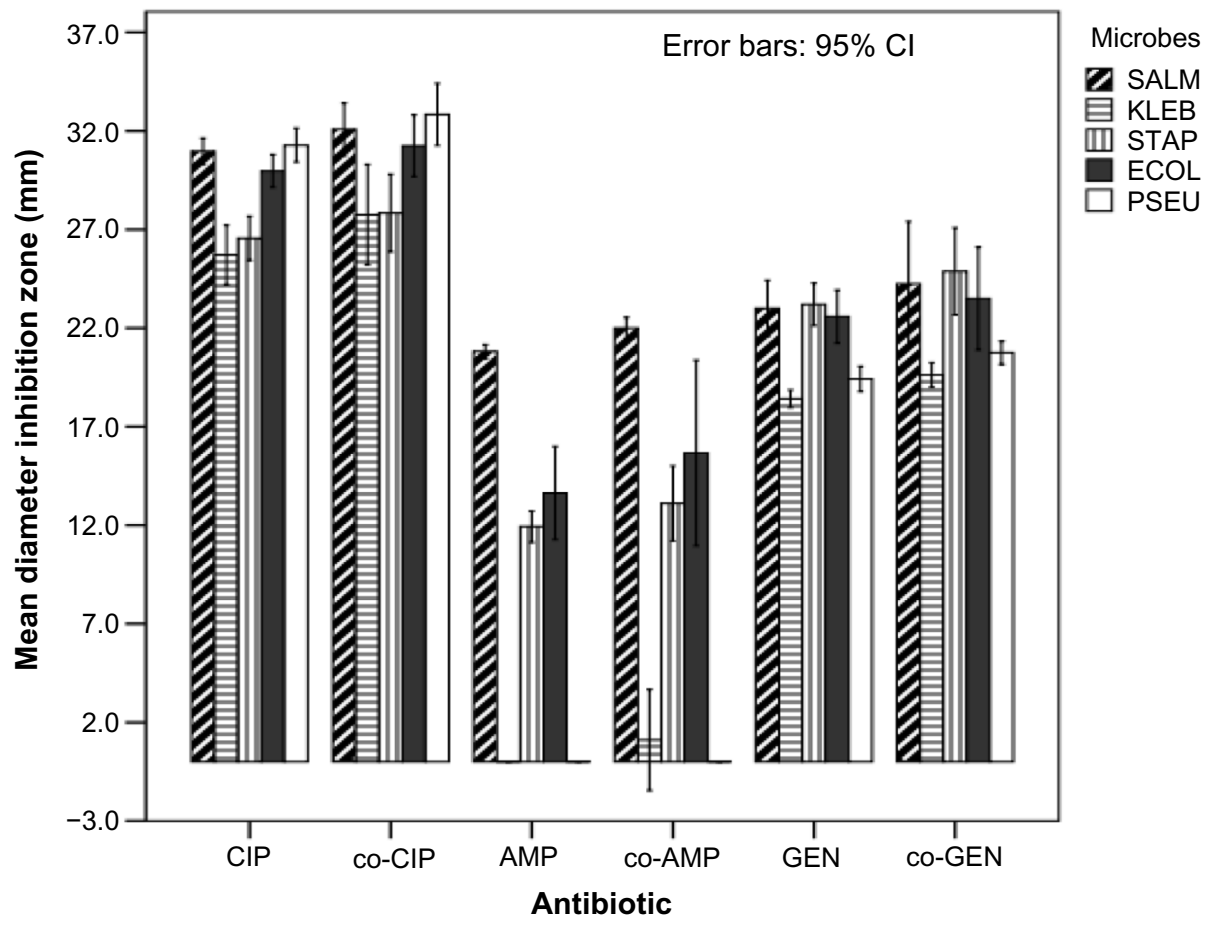

Figure 2 Antibacterial profiles of control antibiotics and test batches of CIP, AMP, and GEN.

Abbreviations: $\mathrm{Cl}$, confidence interval; SALM, Salmonella typhi; KLEB, Klebsiella spp.; STAP, Staphylococcus aureus; ECOL, Escherichia coli; PSEU, Pseudomonas aeruginosa; CIP, ciprofloxacin; co-CIP, control ciprofloxacin; AMP, ampicillin; co-AMP, control ampicillin; GEN, gentamicin; co-GEN, control gentamicin. 


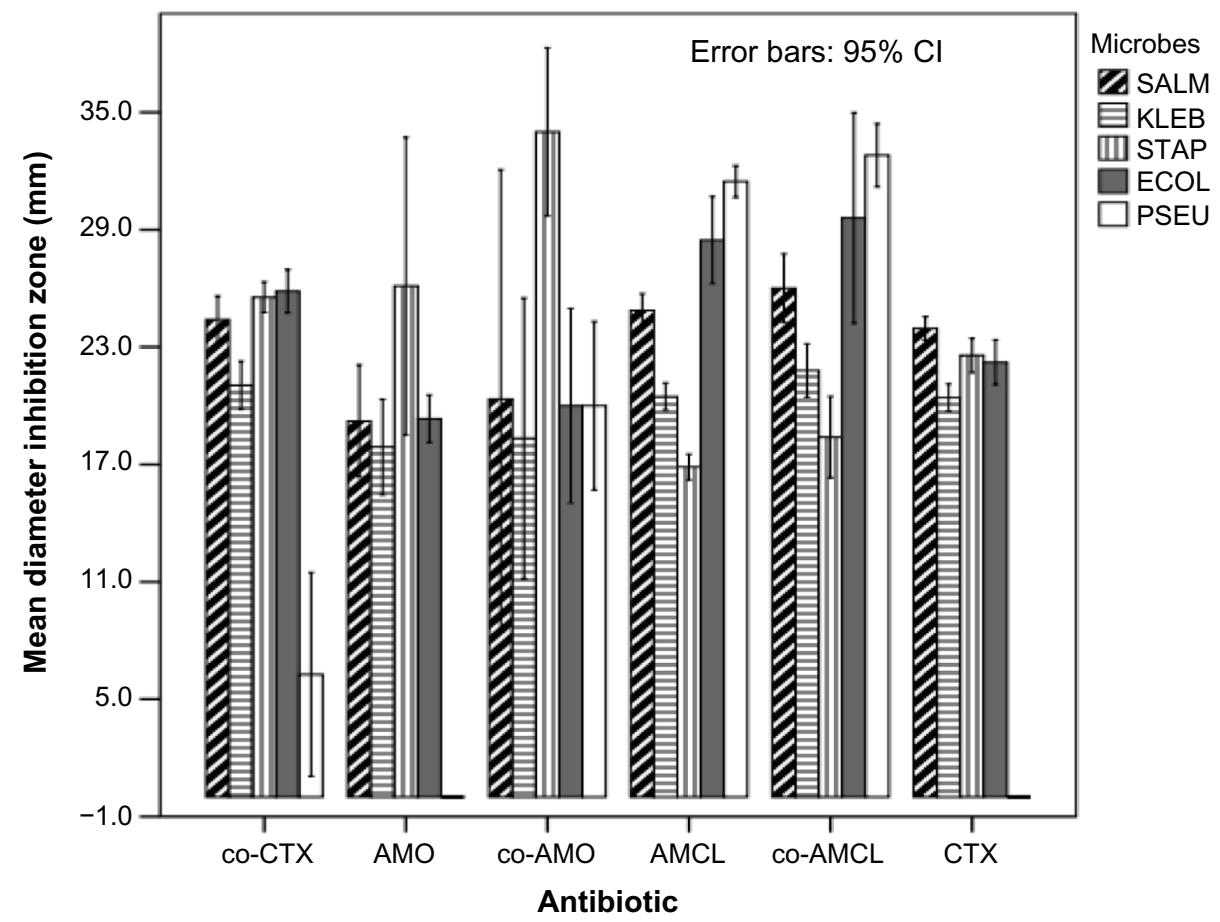

Figure 3 Antibacterial profiles of control antibiotics and test batches of AMO, AMCL, and CTX.

Abbreviations: Cl, confidence interval; SALM, Salmonella typhi; KLEB, Klebsiella spp.; STAP, Staphylococcus aureus; ECOL, Escherichia coli; PSEU, Pseudomonas aeruginosa; co-CTX, control cotrimoxazole; AMO, amoxicillin; co-AMO, control amoxicillin; AMCL, amoxicillin/clavulanate; co-AMCL, control amoxicillin/clavulanate; CTX, cotrimoxazole.

\section{Klebsiella spp.}

Figure 2 depicts a comparative analysis of IZ among 15 different samples of CIP, which revealed significant differences among them $(P=0.001 ; F=23.133 ; d f=14)$. The IZ of samples from two of the five manufacturers (Table 3 ) differed significantly from the control $(P=0.008$ and $P=0.0001)$; these two samples were from Cyprus and India, respectively. Five samples of AMP (Table 3) showed no significant differences in IZ among them $(P=0.469 ; F=1.00 ; d f=4)$. One sample of AMP from India and one from Tanzania differed significantly from the control-AMP $(P=0.019$ and $P=0.006$; respectively). Eight samples of GEN differed significantly $(P=0.01 ; F=4.041 ; d f=7)$ and of these, only three samples were statistically different from the control-GEN $(P=0.004$; $P=0.01$; and $P=0.0001$ ).

The results using AMCL samples were significantly different $(P=0.002 ; F=4.267 ; d f=4)$, whereas only one sample (from Tanzania) showed a significant difference as compared to the control-AMCL $(P=0.005$; Figure 3$)$. Klebsiella spp. were affected differently by the samples of CTX, as significant differences were exhibited among them ( $P=0.0001 ; F=132.605 ; d f=19)$; however, only two CTX samples were significantly different from the control-CTX $(P<0.05)$. Samples of AMO showed significant differences among them $(P=0.0001 ; F=361.00 ; d f=2)$. Only one of the three samples of AMO from India produced significant differences in IZ with respect to control-AMO $(P=0.004)$. Significant differences among samples of ERY were observed $(P=0.0001 ; F=48.956 ; d f=10)$; however, only three of eleven samples of ERY differed significantly from the control-ERY ( $P=0.0001 ; P=0.003$; and $P=0.027$ ). Four samples of the CHR tested exhibited significant differences in IZ among them ( $P=0.001 ; F=248.00 ; d f=3$ ), but only two of them exhibited IZ that differed significantly from the control-CHR $(P<0.01)$. Six samples of TET showed significant differences in their IZ against Klebsiella spp. $(P=0.0001 ; F=20.470 ; d f=5)$, as shown in Figure 4. Of the seven samples, only two samples yielded IZ that were significantly different from that of the control-TET. These samples were from Kenya $(P=0.003)$ and India $(P=0.002)$.

\section{Staphylococcus aureus}

Statistically significant differences in IZ were revealed among samples of CIP $(P=0.001 ; F=46.753 ; d f=14)$. However, only one batch of CIP from an Indian manufacturer produced IZ that differed significantly from that of the control-CIP $(P=0.001)$. Likewise, samples of AMP showed significant differences in IZ among them $(P=0.001 ; F=7.399 ; d f=4)$. Two samples (one from India and one from Kenya) of the nine AMP samples yielded results that were significantly different. 


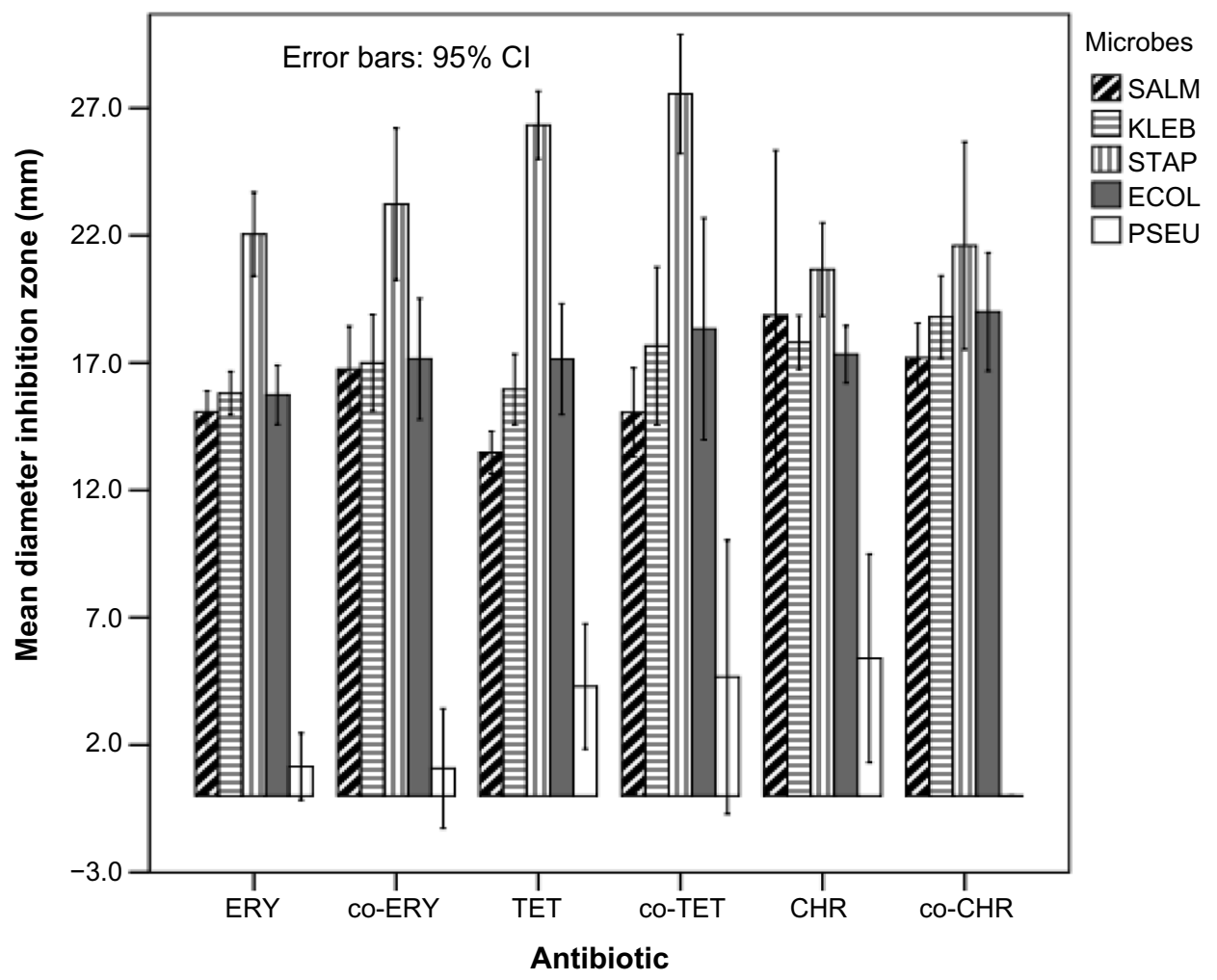

Figure 4 Antibacterial profiles of control antibiotics.

Abbreviations: CI, confidence interval; SALM, Salmonella typhi; KLEB, Klebsiella spp.; STAP, Staphylococcus aureus; ECOL, Escherichia coli; PSEU, Pseudomonas aeruginosa; ERY, erythromycin; co-ERY, control erythromycin; TET, tetracycline; co-TET, control tetracycline; CHR, chloramphenicol; co-CHR, control chloramphenicol.

Significant differences in IZ were also observed among samples of $\operatorname{GEN}(P=0.001 ; F=15.443 ; d f=7)$, although only one sample from Kenya exhibited significant differences as compared to the control-GEN ( $P=0.003)$. The IZ observed using AMCL differed significantly $(P=0.029 ; F=4.227$; $d f=4$ ), for two of the five samples (Table 3 ) showed significant differences in IZ with respect to the control-AMCL $(P<0.05)$. Separately tested samples of CHR, TET, CTX, and ERY displayed IZ that significantly differed within each set of drugs $(P<0.05)$, as shown in Figures 3 and 4 . The results of testing each of the samples with its control antibiotic revealed significant differences: two samples of CHR (one sample from Kenya $[P=0.018]$ and one sample from an anonymous manufacturer $[P=0.024]$ ); one sample of TET from another anonymous manufacturer $(P=0.0001)$; and three samples of ERY (one from India $[P=0.005]$, one from Tanzania $[P=0.002]$, and one from an anonymous manufacturer $[P=0.0001])$. Only one sample of CTX from one of the six manufacturers produced an IZ that was statistically comparable to the control-CTX.

\section{Escherichia coli}

The IZ produced by 15 samples of CIP differed significantly ( $P=0.0001 ; F=25.755 ; d f=14)$; however, only a single sample from India differed significantly from the control-CIP $(P=0.02)$. Samples of AMP showed IZ that were significantly different among them $(P=0.001 ; F=11.053 ; d f=4)$; nevertheless, only one batch of AMP produced IZ that was significantly different from the control-AMP $(P>0.05)$. Separately tested samples of GEN, ERY, TET, and CHR showed IZ that were significantly different among them $(P<0.05)$. The significant differences between the samples and their respective control antibiotics were revealed for one sample of GEN from India $(P=0.013)$ and three samples of ERY (one each from Tanzania, India, and an anonymous producer), exhibiting $P$-values of $0.001,0.0001$, and 0.029 , respectively. Likewise, significant differences between two samples of TET (one from Kenya and one from India) and two samples of CHR (one from Kenya and another from an anonymous source) with their control antibiotics were exhibited (Figures 2-4). The IZ produced by the three samples of AMO against $E$. coli showed statistically significant differences among them $(P=0.005 ; F=15.00 ; d f=2)$. However, no significant differences in IZ were revealed between the samples and the control-AMO $(P>0.05)$, as shown in Figure 3.

\section{Pseudomonas aeruginosa}

Statistically significant differences were observed among samples of each of the following: CIP; AMCL; GEN; ERY; 
TET; and CHR $(P=0.0001 ; P=0.033 ; P=0.0001 ; P=0.0001$; and $P=0.0001$, respectively). Variance was not computed for samples of CTX and AMO, as IZ were not produced; the same significant differences were evident among the three samples of AMO and the control antibiotic $(P=0.0001)$. All samples of AMP produced IZ that were statistically not different from that of the control-AMP $(P>0.05)$, as shown in Figures 2 and 3. Three samples of GEN produced IZ that were significantly different from that of the control-GEN. These three samples were from the People's Republic of China $(P=0.001)$, India ( $P=0.007)$, and Kenya $(P=0.0001)$. Only one sample of AMCL and CIP (both from India) yielded IZ that were statistically different from that of the control antibiotics $(P=0.0001)$. Conversely, no significant differences in IZ were revealed among samples of CHR and TET and their respective control antibiotics $(P>0.05)$.

\section{Discussion}

An abundance of poor quality medicines is related to counterfeiting and/or substandard medicines, poor storage, chemical instability (common in tropical climates), and poor quality control during manufacturing. ${ }^{28,29}$ Although many reasons have been ascribed to the increased prevalence of substandard and counterfeit medications, much of the counterfeit medicines trade seems to be linked to organized crime, corruption, and unregulated pharmaceutical companies. ${ }^{10,30}$ The WHO defines a counterfeit drug as a drug that is "deliberately and fraudulently mislabeled with respect to identity and/or source", and substandard drugs as "genuine drug products which do not meet quality specifications set for them". ${ }^{31}$ Therefore, if substandard drugs are intentionally produced to make an unauthorized product, they too are considered counterfeit.

The present study shows the variability of antibacterial effects of different samples of the same types of antibiotics on reference strains of bacteria. This could be due to differences in formulation of the antibiotics. Such variability could lead to minor, statistically insignificant differences in antibacterial effects. ${ }^{32,33}$ For instance, the differences in tablet compaction forces may, in turn, affect the disintegration of tablets and influence dissolution, which could explain some of the observed differences. However, such differences would be more noticeable in vivo. ${ }^{34,35}$

The present study revealed the existence of two samples of the same type of antibiotic (CIP) with identical batch numbers, but from two different countries. The differences between them include their expiration dates (3 years versus 5 years). Such long expiration dates are unusual for medicines. ${ }^{36}$ A pharmacist at the United States Food and Drug
Administration noted that "Manufacturers put expiration dates on for marketing, rather than scientific reasons ..." 37,38 However, it is difficult for consumers or health care providers to know which product could have an extended shelf life - the expiration date is the final day for which the manufacturer guarantees the full potency and safety of a medication. ${ }^{38,39}$ Antibiotics can be affected by physicochemical changes and environmental conditions. ${ }^{38}$

Our study revealed that 29 samples had neither the manufacturing nor expiration dates, whereas six samples were produced by anonymous manufacturers. This is a health care problem because it is not possible to know how long such drugs are on the market, and their authenticity is therefore questionable. The expiration dates on medicines are often difficult to visualize because of poor legibility, as well as due to their variable location on containers or blisters. ${ }^{40}$ Further, medicines that exceed their expiration dates could be wasted. In Tanzania, several patients reported variability of antibiotic efficacy, which has led some people to be very selective about an antibiotics' country of origin. Such selectivity can only be afforded by the minority of Tanzanians who are financially well-off. This demoralizes patients and negatively affects the medicines/antibiotics available in the market, as well as the health care delivery system at large, because almost $90 \%$ of the medicines are imported using scarce resources. The presence of drugs, particularly antibiotics, without manufacturing or expiration dates is a serious matter and is not an issue of substandard drugs or poor oversight during the manufacturing process, but it is a deliberate act because most patients do not care about expiration dates as longs as the drugs are inexpensive.

CHR has been reported to be ineffective against many pathogenic bacteria, particularly Pseudomonas spp.; nevertheless, our study indicates that CHR remains effective. The inconsistency of CHR effects on Pseudomonas spp. and $S$. typhi raises the question of whether this antibiotic is genuine CHR or a mixture of antibiotics. Otherwise, the positive effects of CHR on the previously reported resistant bacteria could be attributed to the deletion of CHR-resistant genes in the existing bacterial population. Other effects of major concern are the differences in effects of samples of AMO and the control (control-AMO) on Pseudomonas spp. and $S$. aureus. Whereas the CTX-positive control exhibited effects on Pseudomonas spp., the purchased CTX samples could not display any effects. However, the variations in the effects of the tested antibiotics against the reference strains of bacteria are not uncommon because each strain has some "intrinsic resistance" to a particular class of antibiotics. ${ }^{25}$ 
Substandard medicines are those that have not been subjected to or have not passed product quality testing protocols. ${ }^{31,41}$ These testing protocols and product specifications have been published in official pharmacopoeias, including the United States Pharmacopoeia, the European Pharmacopoeia, and the WHO International Pharmacopoeia. In addition, many countries have published their own pharmacopoeias; thus, discrepancies between countries on what constitutes a substandard drug can influence the integrity and availability of drugs in the market. Substandard antibiotics can cause unpredictable treatment results, treatment failure, and promote the development of antibiotic resistance. ${ }^{7,21}$

\section{Conclusion}

The results of this study revealed variability in the effectiveness of antibiotics available in the administrative regions along the borders of Tanzania that were studied. This suggests the presence of substandard and/or counterfeit antibiotics in these regions. In addition, variations in the effectiveness of different samples of the same type of antibiotic were also observed. In the presence of AMP, CHR, TCX, or AMO, bacteria exhibited antibiotic susceptibility patterns that were different from those obtained using the respective control antibiotics. This study did not demonstrate the prevalence of counterfeit antibiotics. However, the results showed that stringent measures are needed to regulate the production and distribution of antibiotics in Tanzania.

\section{Acknowledgments}

The author acknowledges financial support from Muhimbili University of Health and Allied Sciences and extends special appreciation for all Bachelor of Pharmacy students who were involved in the antibiotic samples collection.

\section{Disclosure}

The author reports no conflicts of interest in this work.

\section{References}

1. Goodman C, Kachur SP, Abdulla S, Bloland P, Mills A. Drug shop regulation and malaria treatment in Tanzania - why do shops break the rules, and does it matter?. Health Policy Plan. 2007;22(6):393-403.

2. Verduin-Muttiganzi R, Verduin-Muttiganzi G. Assessment of the incidence of substandard drugs in developing countries. Trop Med Int Health. 1998;3(7):602.

3. Okonko IO, Soleye FA, Amusan TA, Ogun AA, Ogunnusi TA, Ejembi J. Incidence of multi-drug resistance (MDR) organisms in Abeokuta, Southwestern Nigeria. Global J Pharmacol. 2009;3(2):69-80.

4. Okeke IN, Aboderin OA, Byarugaba DK, Ojo KK, Opintan JA. Growing problem of multidrug-resistant enteric pathogens in Africa. Emerg Infect Dis. 2007;13(11):1640-1646.
5. Lahon K, Bairagi KK, Chaturvedi RK. Global scenario in counterfeit medicines: threat assessment, existing remedies and recommendations. Indian Journal of Public Health Research and Development. 2012;3(1).

6. Wertheimer AI, Norris J. Safeguarding against substandard/counterfeit drugs: mitigating a macroeconomic pandemic. Res Social Adm Pharm. 2009;5(1):4-16

7. Abubakar EMM. Antimicrobial susceptibility pattern of pathogenic bacteria causing urinary tract infections at the Specialist Hospital, Yola, Adamawa state, Nigeria. $J$ Clin Med Res. 2009;1(1):1-8.

8. Newton PN, Green MD, Fernández FM, Day NP, White NJ. Counterfeit anti-infective drugs. Lancet Infect Dis. 2006;6(9):602-613.

9. Caudron JM, Ford N, Henkens M, Macé C, Kiddle-Monroe R, Pinel J. Substandard medicines in resource-poor settings: a problem that can no longer be ignored. Trop Med Int Health. 2008;13(8):1062-1072.

10. Kelesidis T, Kelesidis I, Rafailidis PI, Falagas ME. Counterfeit or substandard antimicrobial drugs: a review of the scientific evidence. J Antimicrob Chemother. 2007;60(2):214-236.

11. Liang BA. Fade to black: Importation and counterfeit drugs. Am J Law Med. 2006;32(2-3):279-323.

12. Taylor RB, Shakoor O, Behrens RH, et al. Pharmacopoeial quality of drugs supplied by Nigerian pharmacies. Lancet. 2001;357(9272): 1933-1936.

13. Wan Po AL. Too much, too little, or none at all: dealing with substandard and fake drugs. Lancet. 2001;357(9272):1904.

14. Menkes DB. Hazardous drugs in developing countries. BMJ. 1997; 315(7122):1557-1558.

15. Blomberg B. Antimicrobial Resistance in Bacterial Infections in Urban and Rural Tanzania [dissertation]. Bergen, Norway: University of Bergen; 2007.

16. Levy SB, Marshall B. Antibacterial resistance worldwide: causes, challenges and responses. Nat Med. 2004;10(Suppl 12):S122-S129.

17. Newton PN, Green MD, Fernández, FM. Impact of poor-quality medicines in the 'developing' world. Trends Pharmacol Sci. 2010;31(3): 99-101.

18. Davies J, Davies D. Origins and evolution of antibiotic resistance. Microbiol Mol Biol Rev. 2010;74(3):417-433.

19. Mwambete KD. Irrational antibiotic usage in boarding secondary school settings in Dar es Salaam. East Afri J Public Health. 2009;6(2): 200-204.

20. Fehintola FA. Pre-hospital and prescription use of antibacterial drugs at a secondary health centre in Ibadan, Nigeria. Afri J Pharm Pharmacol. 2009;3(4):120-123.

21. Bonomo RA, Szabo D. Mechanisms of multidrug resistance in Acinetobacter species and Pseudomonas aeruginosa. Clin Infect Dis. 2006;43 Supp1 2:S49-S56.

22. Henrichfreise B, Wiegand I, Pfister W, Wiedemann B. Resistance mechanisms of multiresistant Pseudomonas aeruginosa strains from Germany and correlation with hypermutation. Antimicrob Agents Chemother. 2007;51(11):4062-4070.

23. Hanif M, Mobarak MR, Ronan A, Rahman D, Donovan JJ Jr, Bennish ML. Fatal renal failure caused by diethylene glycol in paracetamol elixir: the Bangladesh epidemic. BMJ. 1995;311(6997):88-91.

24. Shakoor O, Taylor RB, Behrens RH. Assessment of the incidence of substandard drugs in developing countries. Trop Med Int Health. 1997;2(9):839-845.

25. Clinical and Laboratory Standards Institute. Performance Standards for Antimicrobial Susceptibility Testing. 16th Informational Supplement. Wayne, PA: Clinical and Laboratory Standards Institute (CLSI) document M100-S16; 2006.

26. National Committee for Clinical Laboratory Standards. Performance Standards for Antimicrobial Susceptibility Testing: 12th Informational Supplement. Wayne, PA: National Committee for Clinical Laboratory Standards (NCCLS) document M100-S12; 2002.

27. Cheesbrough M. Antimicrobial sensitivity testing, in District Laboratory Practice in Tropical Countries. Cambridge, UK: Cambridge University Press; 2006 
28. Hogerzeil HV, de Goeje MJ, Abu-Reid IO. Stability of essential drugs in Sudan. Lancet. 1991;338(8769):754-755.

29. Arya SC. Inadvertent supply of substandard drugs. World Health Forum. 1995; 16(3):269.

30. Moken MC. Fake pharmaceuticals: how they and relevant legislation or lack thereof contribute to consistently high and increasing drug prices. Am J Law Med. 2003;29(4):525-542.

31. World Health Organization [webpage on the Internet] General information on counterfeit medicines [updated 2014; cited May 23, 2014]. Geneva Switzerland: World Health Organization; 2014. Available from: http:// www.who.int/medicines/services/counterfeit/overview/en. Accessed May 23, 2014.

32. Late SG, Yu YY, Banga AK. Effects of disintegration-promoting agent, lubricants and moisture treatment on optimized fast disintegrating tablets. Int J Pharm. 2009;365(1-2):4-11.

33. Jivraj M, Martini LG, Thomson CM. An overview of the different excipients useful for the direct compression of tablets. Pharm Sci Technol Today. 2000;3(2):58-63.

34. Holford, NHG. Rational dosing and time course of drug action. In: Katzung BG, Masters SB, Trevor AJ, editors. Pharmacokinetics and Pharmacodynamics. 11th ed. San Francisco: McGraw-Hill Companies; 2009:34-50
35. Marais AF, Song M, de Villiers MM. Effect of compression force, humidity and disintegrant concentration on the disintegration and dissolution of directly compressed furosemide tablets using croscarmellose sodium as disintegrant. Trop J Pharm Res. 2003;2(1):125-135.

36. Ekedahl AB. Reasons why medicines are returned to Swedish pharmacies unused. Pharm World Sci. 2006;28(6):352-358.

37. Ette EI. Conscience, the law, and donation of expired drugs. Ann Pharmacother. 2004;38(7-8):1310-1313.

38. Lyon RC, Taylor JS, Porter DA, Prasanna HR, Hussain AS. Stability profiles of drug products extended beyond labeled expiration dates. J Pharm Sci. 2006;95(7):1549-1560.

39. [No authors listed]. Drugs past their expiration date. Med Lett Drugs Ther. 2009;51:100-101

40. Lohiya S. A simple stratification and storage system for samples and supplies. J Natl Med Assoc. 2006;98(3):405-407.

41. Cohen JC, Mrazek M, Hawkins L. Tackling corruption in the pharmaceutical systems worldwide with courage and conviction. Clin Pharmacol Ther. 2007;81(3):445-449.
Integrated Pharmacy Research and Practice

\section{Publish your work in this journal}

Integrated Pharmacy Research and Practice is an international, peer-reviewed, open access, online journal, publishing original research, reports, reviews and commentaries on all areas of academic and professional pharmacy practice. This journal aims to represent the academic output of pharmacists and pharmacy practice with particular focus on integrated care. All papers are carefully peer reviewed

\section{Dovepress}

to ensure the highest standards as well as ensuring that we are informing and stimulating pharmaceutical professionals. The manuscript management system is completely online and includes a very quick and fair peer-review system, which is all easy to use. Visit http://www.dovepress.com/testimonials.php to read real quotes from published authors.

Submit your manuscript here: http://www.dovepress.com/integrated-pharmacy-research-and-practice-journal 